Special issue of the 2nd International Conference on Computational and Experimental Science and Engineering (ICCESEN 2015)

\title{
The Trap of Dust Particles with Different Sizes in Electronegative Plasma Sheaths
}

\author{
D. Benlemdjaldi ${ }^{a, b, *}$, A. Tahraoui ${ }^{b}$, N. Fouial ${ }^{b}$ And S. Chekour ${ }^{b}$ \\ ${ }^{a}$ Ecole Normale Supérieure, Département de Physique, BP 92 Vieux-Kouba, 16050 Algiers, Algeria \\ ${ }^{b}$ USTHB, Faculty of Physics, PB 32, El-Alia, 16111, Bab-Ezzouar, Algiers, Algeria
}

\begin{abstract}
In this work, we have examined the dynamics of dust particles in the electronegative plasma sheath. For this, we have developed a 1D stationary and unmagnetized model. The electrons and the negative ions are considered in thermodynamic equilibrium, while the positive ions and the impurities (dust grains) are described by fluid equations model. The impurities are considered to have spherical forms; moreover their size distribution is given by Gaussian distribution. Several forces acting on the dust particles are taken into account. The numerical results show that the contribution of the neutral drag force is negligible compared to others forces. In addition, the electrostatic force acting on the $\mathrm{nm}$ particles is dominant. Also, we found that the suspension of dust grains in electrostatic sheath is only for a small dust radius interval for which the electrostatic force is balanced by the gravity and the ion drag forces.
\end{abstract}

DOI: 10.12693/APhysPolA.130.11

PACS/topics: 52.25.Vy, 52.27.Lw, 52.40.Kh

\section{Introduction}

The interaction of plasmas with solid surfaces has received great attention over the last three decades in order to understand many physical phenomena such as surface erosion, plasma processing, and ion cyclotron heating. This interaction gives rise to a non-neutral region called electrostatic sheath [1] where a strong localized electric field is formed due to the high mobility of electrons in contrast to ions mobility. The typical sheath thickness is a few electron Debye lengths, which is usually small compared with the characteristic length of the plasma defined by the typical size of the system. Nonetheless, the presence of dust grains ranging from $\mathrm{nm}$ to $\mu \mathrm{m}$ in size leads to important modifications in the sheath behavior and therefore remains an important and serious problem in plasma processing of integrated circuits for example $[2,3]$. Several researchers have investigated analytically and experimentally the effect of dust grains in the electrostatic sheath behavior $[4,6]$. In this paper, we have examined the trap of dust particles in the electronegative plasma sheath.

\section{Theoretical model}

We consider a $1 \mathrm{D}$, stationary and unmagnetized dusty plasma consisting of electrons $(e)$, negative ions $(j)$, positive ions $(i)$ and dust grains $(d)$ with different sizes. We assume that the vertical axis has the same direction as gravity, then $z<0$ is the plasma region and $z>0$ is the sheath region. At the edge $z=0$, we assume the electrostatic potential $\phi=0$, and the number density of specie $l(l=e, j, i, d)$ is $n_{l 0}$. The subscript " 0 " denotes the equilibrium quantities $(\phi=0)$. The electrons and negative ions are assumed to be in thermal equilibrium, thus

\footnotetext{
* corresponding author; e-mail: dbenlemdjaldi@gmail.com
}

there number densities $n_{e}$ and $n_{j}$ satisfy the Boltzmann relation

$$
n_{e, j}=n_{e 0, j 0} \exp \left(\frac{e \phi}{T_{e, j}}\right),
$$

where $T_{e, j}$ is the temperature of specie $(e, j)$. The positive ions are described by fluid equations

$$
\begin{aligned}
& \frac{\mathrm{d}\left(n_{i} v_{i}\right)}{\mathrm{d} z}=k_{i} n_{e} n_{\mathrm{n}}, \\
& v_{i} \frac{\mathrm{d} v_{i}}{\mathrm{~d} z}=-\frac{e}{m_{i}} \frac{\mathrm{d} \phi}{\mathrm{d} z}-\frac{1}{m_{i} n_{i}} \frac{\mathrm{d} p_{i}}{\mathrm{~d} z}-\frac{F_{\text {in }}}{m_{i}},
\end{aligned}
$$

where $k_{i}$ is the electronic impact ionization rate. $n_{i}, v_{i}$, and $p_{i}$ are the density, the entering sheath fluid velocity toward to the wall, and pressure of the positive ions respectively, $n_{\mathrm{n}}$ is the neutral gas density, which is considered constant, $F_{\mathrm{in}}=m_{i} n_{\mathrm{n}} \sigma_{\mathrm{in}} v_{i}^{2}$ is a drag force due to collisions with neutrals, $\sigma_{\text {in }}$ being the ion-neutral cross-section. We assume that the ions flow is adiabatic. For unidimensional problem we have $P_{i}=\frac{T_{i}}{n_{i 0}^{2}} n_{i}^{3}$.

The dust grains with different sizes are also described by the continuity and momentum equations

$$
\begin{aligned}
& \frac{\mathrm{d}\left(n_{d k} v_{d k}\right)}{\mathrm{d} z}=0, \\
& m_{d k} v_{d k} \frac{\mathrm{d} v_{d k}}{\mathrm{~d} z}=f_{e k}+f_{g k}+f_{n d k}+f_{i d k},
\end{aligned}
$$

where $n_{d k}, q_{d k}, m_{d k}$ and $v_{d k}$ are respectively the number density, the charge, the mass and the fluid velocity of $k$-th dust grains which are considered spherical with radius $r_{d k}$.

The main forces acting on the $k$-th dust grains are electrostatic force $f_{e k}=-q_{d k} \frac{\mathrm{d} \phi}{\mathrm{d} z}$, gravitational force $f_{g k}=\frac{4}{3} \pi r_{d k}^{3} \rho_{d} g$, neutral drag force [7]

$$
f_{n k}=-\frac{8}{3}(2 \pi)^{1 / 2} \frac{r_{d k}^{2} n_{\mathrm{n}} T_{\mathrm{n}}}{v_{t n}}\left(v_{d k}-v_{\mathrm{n}}\right),
$$

and ion drag force $[8]$ 
$f_{i d k}=\pi n_{i} m_{i} v_{i t} v_{i}\left(b_{c k}^{2}+2 b_{(\pi / 2) k}^{2} \ln \left(\frac{\lambda_{\mathrm{D}}^{2}+b_{(\pi / 2) k}^{2}}{b_{c}^{2}+b_{(\pi / 2) k}^{2}}\right)\right)$.

$b_{c k}=r_{d k}\left(1-2 e q_{d k} / m_{i} v_{i t}^{2} r_{d k}\right)^{1 / 2}$ is the collection impact parameter, $b_{(\pi / 2) k}=e q_{d k} / m_{i} v_{i t}^{2}$ is the orbital impact parameter $v_{i t}=\left(v_{i}^{2}+8 T_{i} / \pi m_{i}\right)^{1 / 2}$ is the total ion velocity, $\lambda_{\mathrm{D}}$ is the generalized Debye length.

The dust grain charge is due only to electron, negative ion and positive ion fluxes arriving on the dust grain, thus, the charge current balance equation for $k$-th dust grain is

$$
v_{d k} \frac{\mathrm{d} q_{d k}}{\mathrm{~d} z}=I_{e k}+I_{i k}+I_{j k}
$$

where $I_{l k}(l=e, i, j)$ denotes the charge currents which are computed by using the orbit motion limited model (OML) [9].

The formulation is completed with the Poisson equation $[10,11]$

$$
\frac{\mathrm{d}^{2} \phi}{\mathrm{d} z^{2}}=-\frac{1}{\varepsilon_{0}}\left(n_{i} e-n_{e} e-n_{j} e+\int_{r_{d \min }}^{r_{d \max }^{\max }} q_{d} \mathrm{~d} n_{d}\right),
$$

where the dust grains number density distribution $\mathrm{d} n_{d}\left(r_{d}\right)$ is given by $\mathrm{d} n_{d}=n_{d} f\left(r_{d}\right) \mathrm{d} r_{d} \cdot r_{d \text { min }}$ and $r_{d \max }$ being the lower and higher values of $r_{d}$, otherwise the distribution function has zero value.

In our case (laboratory plasmas), the most used dust size distribution function is a Gaussian one defined

$$
f\left(r_{d}\right)=D_{g} \exp \left(-\mu\left(r_{d}-r_{d m}\right)^{2}\right),
$$

where $r_{d m}$ is the average radius of dust size, $D_{g}$ and $\mu$ are constants [10].

For numerical simulation, the previous equations are converted into dimensionless from using the following normalized variables:

$$
\begin{aligned}
& \xi=z / \lambda_{\mathrm{D} i}, \quad \Phi=-e \phi / T_{e}, \quad N_{e}=n_{e} / n_{e 0}, \\
& N_{i}=n_{i} / n_{i 0}, \quad u_{i}=v_{i} / c_{i s}, \quad \Phi_{d}=e q_{d} / r_{d} T_{e} \\
& N_{d}=n_{d} / n_{d 0}, \quad u_{d 0}=v_{d 0} / c_{d s m}, \quad \gamma_{i}=T_{e} / T_{i}, \\
& \gamma_{j}=T_{j} / T_{i}, \quad \delta_{e m}=n_{e 0} / n_{i o}, \quad \delta_{d}=n_{d 0} / n_{i o} \\
& F_{l k}=f_{l k} / f_{0}
\end{aligned}
$$

where

$$
\begin{aligned}
& f_{0}=z_{c m} T_{e} / \lambda_{\mathrm{D} i}, \quad \phi_{d}=q_{d k} / r_{d k} \\
& c_{d s m}=\left(z_{c m} T_{e} / m_{d m}\right)^{1 / 2}, \quad z_{c m}=r_{d m} T_{e} / e^{2}, \\
& m_{d m}=\rho_{d} \frac{4}{3} \pi r_{d m}^{3} .
\end{aligned}
$$

\section{Results and discussion}

In this section, we present the numerical results of our theoretical model. We have implement our model by considering an oxygen plasma with typical parameters of electronegative dusty plasmas, $T_{e}=2 \mathrm{eV}, T_{i}=$ $T_{j}=T_{\mathrm{n}}=0.05 \mathrm{eV}, P_{\mathrm{n}}=1 \mathrm{mTorr}, n_{i 0}=10^{9} \mathrm{~cm}^{-3}$, $\sigma_{\text {in }}=10^{-14} \mathrm{~cm}^{2}, \rho_{d}=2 \mathrm{~g} / \mathrm{cm}^{3}, \delta_{d}=10^{-4}$ and $u_{i 0}=1.5$; $k_{i}=10^{-11} \mathrm{~cm}^{3} / \mathrm{s}, r_{d \min }=0.01 \mu \mathrm{m}$ and $r_{d \max }=4.5 \mu \mathrm{m}$. The negative and positive ions are $\mathrm{O}_{2}^{-}$and $\mathrm{O}_{2}^{+}$. The normalized initial conditions are summarized as follows:

$$
\begin{aligned}
& \Phi(\xi=0)=0, \quad \frac{\mathrm{d} \Phi}{\mathrm{d} \xi}(\xi=0)=\alpha_{c i} u_{i 0}^{2}+\frac{3 k_{i} n_{\mathrm{n}} \lambda_{\mathrm{D} i} \delta_{e}}{\gamma_{i} c_{i s} u_{i 0}} \\
& N_{i}(\xi=0)=1, \quad \Psi(\xi=0)=0, \quad \Phi_{d}(\xi=0)=\Phi_{d 0}, \\
& \Omega\left(\xi=0, r_{d}=\alpha_{1}\right)=0, \quad \Omega\left(\xi=0, r_{d}=\alpha_{2}\right)=0, \\
& \frac{\mathrm{d} u_{i}}{\mathrm{~d} \xi}(\xi=0)=0 .
\end{aligned}
$$

The density ratio of the electron to the ion equilibrium is computed from the quasi-neutrality equation and the dust grain surface potential at equilibrium $\Phi_{d}(\xi=0)=$ $\Phi_{d 0}$ is computed numerically from the charge conservation $I_{e}+I_{j}+I_{i}=0$.
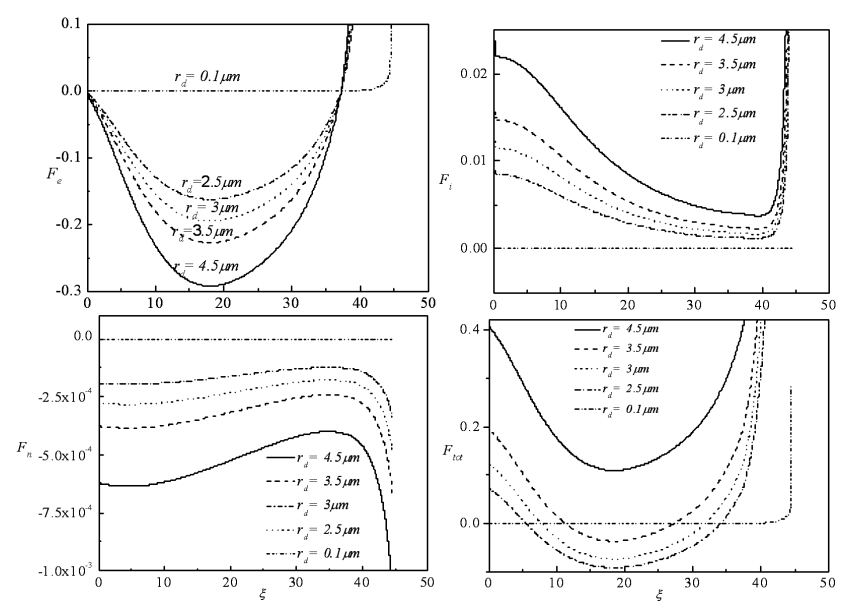

Fig. 1. Normalized electrostatic force $F_{e}$, normalized ion drag force $F_{i}$, normalized neutral drag force $F_{\mathrm{n}}$, and net force acting on dust grain $F_{\text {tot }}$ versus normalized position $\xi$.

Figure 1 shows the different forces acting on a specific dust grain with constant radius. First, we observe that the contribution of the neutral drag force acting on the dust grains is negligible compared to others forces. Moreover, the contribution of the gravity force is also negligible for $\mathrm{nm}$ particles. However, for $\mu \mathrm{m}$ particles, its contribution is important compared to the electrostatic and the ion drag forces. According to our model, the sign of the gravity and the ion drag forces is always positive. However, the electrostatic force is positive only in the electrode vicinity. We observe also that the trap of dust grains in electrostatic sheath is only possible for a small dust radius interval for which the electrostatic force is balanced by the gravity and the ion drag forces. This dust radius interval is around the most probable dust radius. 


\section{References}

[1] M.A. Lieberman, A.J. Lichtenberg, Principles of Plasma Discharges and Materials Processing, Wiley, New York 2005.

[2] L.I. Ryabova, D.R. Khokhlov, JETP Lett. 80, 133 (2004).

[3] S.G. Ovchinnikov, Zh. Eksp. Teor. Fiz. 102, 534 (1992).

[4] D. Benlemdjaldi, A. Tahraoui, R. Hugon, J. Bougdira, Phys. Plasmas 20, 043508 (2013).

[5] G. Foroutan, A. Akhoundi, Phys. Plasmas 19, 103505 (2012).
[6] S. Dap, D. Lacroix, R. Hugon, L. de Poucques, J.L. Briancon, J. Bougdira, Phys. Rev. Lett. 109, 245002 (2012).

[7] M.S. Barnes, J.H. Keller, J.C. Forster, J.A. O'Neill, D.K. Coultas, Phys. Rev. Lett. 68, 313 (1992).

[8] P.S. Epstein, Phys. Rev. 23, 710 (1924).

[9] A. Tahraoui, B. Zaham, R. Annou, J. Bougdira, R. Hugon, AIP Conf. Proc. 1047, 268 (2008).

[10] W.S. Duan, H.J.Y.R. Shi, K.P. Lv, Phys. Lett. A 361, 368 (2007).

[11] O. Havnes, T.K. Aanesen, F. Melandso, J. Geophys. Res. 95, 6581 (1990). 